\title{
Accessory mitral valve misdiagnosed as vegetation-A case report
}

\author{
Rezvanieh Salehi ${ }^{1}$, Naser khezerlouy aghdam ${ }^{1}$, Kamran Mohammadi ${ }^{1}$, and Babak Zanjani ${ }^{1}$ \\ ${ }^{1}$ Tabriz University of Medical Sciences Faculty of Medicine
}

May 27, 2020

\begin{abstract}
Abstract Accessory mitral valve (AMV) is a rare anomaly of mitral valve which often causes left ventricular outflow tract (LVOT) obstruction. Different congenital anomalies, including bicuspid aortic valve, are associated with this rare anomaly. Distinguishing AMV from other mitral valve masses is sometimes difficult and can lead to unnecessary diagnostic and therapeutic measures. We present a case report regarding the AMV without LVOT obstruction primarily misdiagnosed as vegetation in an adult. Keywords: Accessory Mitral Valve, Vegetation, Mass
\end{abstract}

\section{Accessory mitral valve misdiagnosed as vegetation-A case report Background:}

Congenital abnormalities of mitral valve include mitral stenosis, double orifice mitral valve, mitral valve parachute, and mitral valve cleft. ${ }^{1}$ Accessory mitral valve () is a rare anomaly of mitral valve, which mostly results in left ventricular outflow tract (LVOT) obstruction. ${ }^{2-5}$ We present a case report regarding the without significant LVOT obstruction primarily misinterpreated as vegetation in an adult.

\section{Case report:}

The patient was a 19 years old woman who referred to advanced echocardiography lab due to a history of heart murmur and a mass attached to anterior mitral leaflet at previous transthoracic echocardiography. Patient didn't have fever, anorexia, weakness, and peripheral stigmata of infective endocarditis or history of emboli. After first echocardiography, patient had been evaluated for infective endocarditis and non-bacterial thrombotic endocarditis (NBTE). All laboratory findings including erythrocyte sedimentation rate (ESR), Creactive protein $(\mathrm{CRP})$, complete blood count $(\mathrm{CBC})$, blood cultures, renal function tests, and screening test for Systemic lupus erythematosus were normal. Ultrasonography of abdomen and pelvic were normal. She underwent transthoracic and transesophageal echocardiography at our center which showed a large mobile oval shape accessory mitral valve, measured about $18 \mathrm{~mm}$ that attached to ventricular surface of the anterior mitral leaflet (Movie clips 1,2). AMV was echogenic, with echo-free central part (cyst-like) and sharp borders which resembles the mitral valve (Figures 1,2). LVOT evaluation with color doppler showed turbulent flow without significant stenosis (mean pressure gradient $=13 \mathrm{mmHg}$, peak pressure gradient $=22 \mathrm{mmHg}$ ). Aortic valve was bicuspid with moderate aortic regurgitation without stenosis. There was a raphe between left coronary cusp (LCC) and right coronary cusp (RCC). Ascending aorta diameter was normal. Evaluation for coarctation of aorta was negative. Mitral valve function was normal without stenosis or regurgitation. At examination, it was a blowing type diastolic murmur along with ejection sound and midsystolic murmur grade II at upper left sternal border (LSB). Despite these findings, patient was asymptomatic.

\section{Discussion:}

The differential diagnosis of mitral valvular masses includes, infective endocarditis, non-bacterial thrombotic endocarditis, organized thrombus, tumoral mass such as papillary fibroelastoma, myxoma, calcified tooth paste tumor of mitral annulus and blood cyst. ${ }^{6}$ Some lesions have distinctive features and can be diagnosed 
easily. Both Myxoma and papillary fibroelastoma have such echocardiographic findings including narrow stalk with mixed echogenic pattern in myxoma or round head with fine shimmering papilla in papillary fibroelastoma. On the other hand some mass have no specific feature at echocardiography and definite diagnosis is impossible especially when echocardiography acoustic window is suboptimal. This uncertainty results in wide differential diagnosis and mandates extensive evaluation with laboratory and other imaging tests. This patient had a bicuspid aortic valve with suspicious mobile mass which misinterpreted as endocarditis and was evaluated for both infective and non-bacterial thrombotic endocarditis. Many congenital heart diseases have multiple different presentations and sometimes have complex lesions. It is important to consider these concomitant lesions at any patient with congenital heart disease to prevent unnecessary measures and costs. According to literature, most case reports described LVOT obstruction and significant stenosis due to the accessory mitral valve, which led to surgical resection. ${ }^{2-5}$ Despite these findings, our patient didn't have significant stenosis at LVOT and was asymptomatic.

\section{Conclusion:}

Accessory mitral valve is a rare congenital heart anomaly. In echocardiography, it is seen as mitral valve mass and can present with or without significant LVOT stenosis. AMV is seen mostly with other congenital anomalies such as bicuspid aortic valve.

1. Anirban Banerjee, Thomas Kohl, Norman H. Silverman. Echocardiographic evaluation of congenital mitral valve anomalies in children. Am J Cardiol. 1995;76(17):1284-1291.

2. Gurzun MM, Husain F, Zaidi A, et al. Accessory Mitral Valve-An Unexpected Intra-Operative TEE Finding Causing Left Ventricular Outflow Tract Obstruction in an Adult. Echocardiography. 2013;31(2):E55-7. doi: 10.1111/echo.12436

3. W G Meldrum-Hanna, T B Cartmill, R E Hawker, et al. Accessory mitral valve tissue causing left ventricular outflow tract obstruction. Br Heart j. 1986;55:376-80. doi: 10.1136/hrt.55.4.376

4. Takashi Tennichi, Takumi Taniguchi. Accessory mitral valve tissue that caused a left ventricular outflow tract obstruction: a case report. JA Clinical Reports. 2019;5:86. doi.org/10.1186/s40981-019-0306-2

5. Shi-Min Yuan, Amihay Shinfeld, David Mishaly, et al. Accessory Mitral Valve Tissue: A Case Report and an Updated Review of Literature. J Card Surg. 2008;23(6):769-72. doi: 10.1111/j.15408191.2008.00633.x

6. Shi-Min Yuan, Hua Jing, Jacob Lavee. Tumors and tumor-like lesions of the heart valves. Rare Tumors. 2009;28;1(2): e35. doi: 10.4081/rt.2009.e35Figures and movie clips legend :Figure 1: Long axis view $\left(143^{\circ}\right)$ at transesophageal echocardiography showed large cyst like mass at ventricular side of anterior mitral leaflet during systole. Figure 2: Long axis view $\left(120^{\circ}\right)$ at transesophageal echocardiography showed large cyst like mass at ventricular side of anterior mitral leaflet during systole. Movie clip 1: Transesophageal echocardiography showed large mobile cyst like mass attached to anterior mitral leaflet Movie clip 2: Transthoracic echocardiography showed large mobile mass attached to anterior mitral leaflet protrude to left ventricle outflow tract during systole 

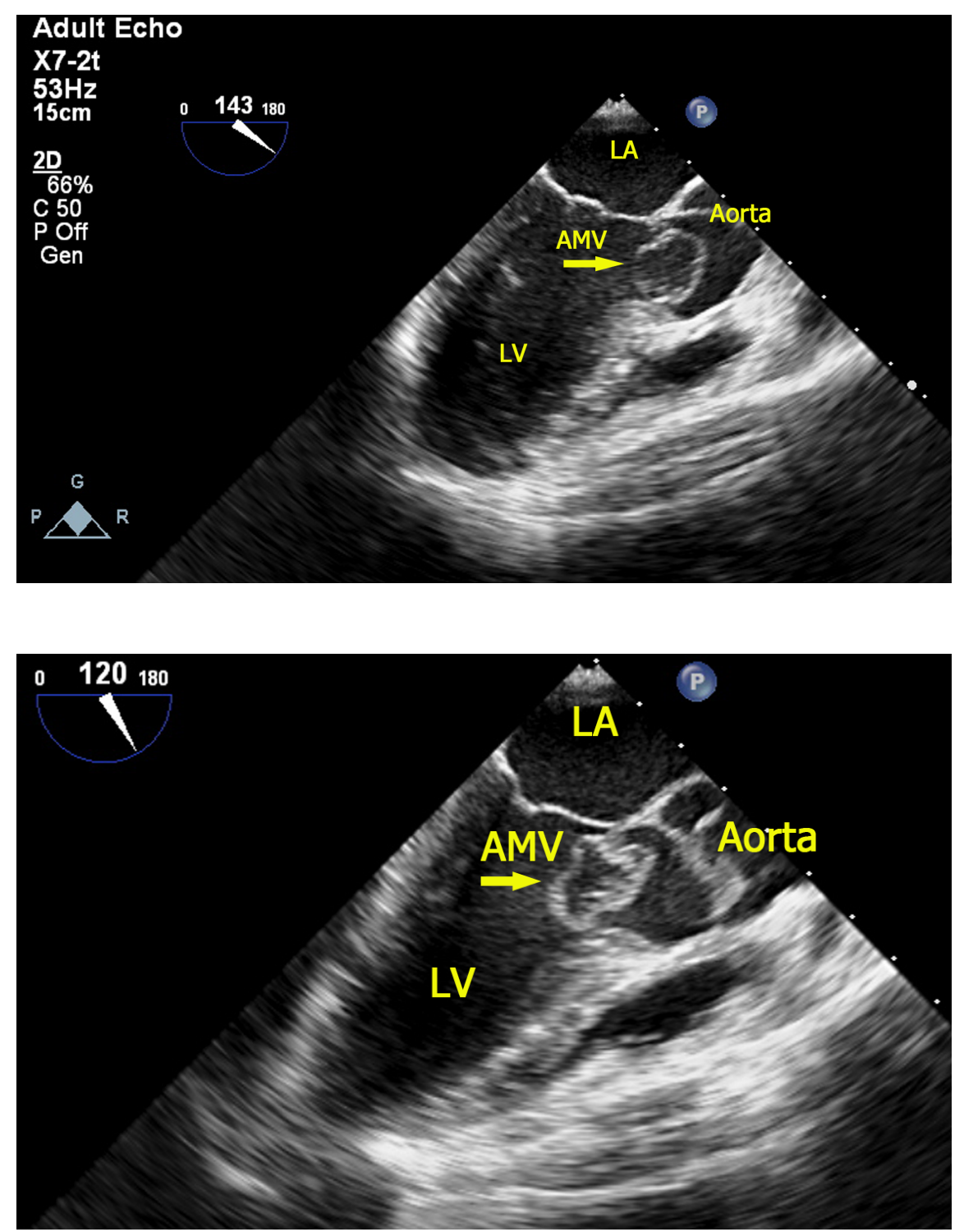